by Jim W. Kennedy ${ }^{*}$, Andy S. Gale ${ }^{2}$, Brian T. Huber ${ }^{3}$, Maria R. Petrizzo $^{4}$, Paul Bown $^{5}$, and Hugh C. Jenkyns ${ }^{6}$

\title{
The Global Boundary Stratotype Section and Point (GSSP) for the base of the Albian Stage, of the Cretaceous, the Col de Pré-Guittard section, Arnayon, Drôme, France
}

\author{
${ }^{1}$ Oxford University Museum of Natural History, Parks Road, Oxford OX1 3PW, UK; *Corresponding author, E-mail: Jim.Kennedy@oum.ox.ac.uk \\ ${ }^{2}$ Department of Earth and Environmental Sciences, University of Portsmouth, Portsmouth POI 3QL, UK \\ ${ }^{3}$ Department of Paleobiology, National Museum of Natural History, Smithsonian Institution, P.O. Box 37012, MRC 121, Washington D.C. \\ 20013-7012, USA \\ ${ }^{4}$ Dipartimento di Scienze della Terra "Desio”, Universita degli Studi di Milano, Via Mangiagalli 34, 20133 Milano, Italy \\ ${ }^{5}$ Department of Earth Sciences, University College London, Gower Street, London WC1E 6BT, UK \\ ${ }^{6}$ Department of Earth Sciences, University of Oxford, South Parks Road, Oxford OX1 3AN, UK
}

(Received: June 3, 2016; Revised accepted: November 29, 2016)

http://dx.doi.org/10.18814/epiiugs/2017/v40i3/017021

Following the unanimous approval of the Executive Committee on the International Union of Geological Sciences as notified on April 8, 2016, the Global boundary Stratotype Section and Point for the base of the Albian Stage of the Cretaceous is defined at the first occurrence datum of the planktonic foraminiferan Microhedbergella renilaevis Huber and Leckie, 2011 at a level 37.4 meters above the base of the Marnes Bleues Formation and 40 cm above the base of the Niveau Kilian marker bed in the section SSE of the Col de Pré-Guittard, Arnayon, Drôme, France. The first occurrence of Microhedbergella renilaevis is placed within a 100-m section of argillaceous sediments with 28 secondary markers including calcareous nannofossils, planktonic foraminifera, an inoceramid bivalve, ammonites, stable carbon isotopes, and local marker beds.

\section{Introduction}

The present document defining a Global boundary Stratotype Section and Point for the base of the Albian Stage of the Lower Cretaceous arises from one of the recommendations of the Albian Working Group of the Subcommission on Cretaceous Stratigraphy at its meetings during the Second International Symposium on Cretaceous Stage boundaries held in Brussels from September 8-15, 1995 (Hart et al., 1996), and the subsequent publications of Petrizzo et al. $(2012,2013)$ and Kennedy et al. (2014).

\section{Historical Background}

The Étage Albien was introduced by Alcide d'Orbigny in 1843 (in
d'Orbigny, 1842-3, p.404), as follows:

"Gault. L'étage ainsi nommée de ses argiles varie on ne peut d'avantage sous le rapport minéralogique. II est en effet forme d'argiles, à ses parties moyennes, à Wissant (Pas-de-Calais), aux Côtes Noires (Haute-Marne), à Gaty, à Maurepaire, à Dienville (Aube), et à Folkestone (Angleterre); mais à Wissant même, à Ervy (Aube); à Saint-Florentin (Yonne), à la pêrte du Rhône (Ain), à Macheromenil (Ardennes), à Varennes (Meuse), il est aussi composé de grès verts, de grès blanchatres; à Escragnolle (Var), il est représenté par une véritable glauconie crayeuse; à la Montagne-des-Fis (Savoie), par des roches noirâitres compactes. On voit donc que les noms de gault, de glauconie sableuse, de grès vert inférieur, ne peuvent non plus être proprément appliqués dans tous les cas, ce qui me détermine à proposer, pour cet étage, le nom de terrain ALBIEN, 1'Aube (Alba) le traversant à Dienville et sur beaucoup d'autres points".

The succession in Aube was carefully documented by Rat et al. (1979), Amédro et al. (1995) and Colleté (2010). Although of considerable historic interest, the area is unsuitable for defining the base of the stage in contemporary terms. As Amédro et al. (1995, p. 34) note, "1'Albien type reste incomplétement connu. Cette situation est liée à 1'absence des coupes continues et à 1'importance de la couverture végétale qui rend les affleurements très rares et éphémères."

It is this lack of suitable permanent sections that makes Aube unsuitable as a location for a GSSP. Furthermore, the lowest fossiliferous Albian recognized is a condensed phosphatic nodule bed at the top of the Sables Verts de 1'Aube (Amédro et al., 1995), which has yielded Hypacanthoplites milletioides Casey, 1961, H. milletianus (d'Orbigny, 1841), Leymeriella (L.) tardefurcata (d'Orbigny, 1841), L. (N.) regularis (d'Orbigny, 1841), and Douvilleiceras mammilatum (Schlotheim, 1813). The underlying Sables Verts have not yielded diagnostic fossils, and rest unconformably on Aptian Argiles à Plicatules (Rat et al., 1979; Amédro et al., 1995).

At the conclusion of the meeting of the Working Group on the 
Albian Stage, held during the Second International Symposium on Cretaceous Stage boundaries, held in Brussels from September 8-16, 1995 (Hart et al., 1996), the succession at the Col de Pré-Guittard, Arnayon, Drôme, France was discussed, and a number of possible palaeontological markers for the boundary noted:

- The first occurrence of the ammonite Leymeriella tardefurcata (d'Orbigny, 1841)

- the first occurrence of the ammonite Douvilleiceras ex gr. mammilatum (Schlotheim, 1813)

- the first occurrence of the coccolithophore Prediscosphaera columnata (Stover, 1966)

- the last occurrence of the ammonite Hypacanthoplites jacobi (Collet, 1907)

- the top or bottom of the Paquier 'oceanic anoxic event'

- the topmost organic-rich bed of the faisceau Kilian

- or "any other datum" (Hart et al., 1996, p. 51).

The first option was pursued by Kennedy et al. (2000), but eventually failed to find favour with the Cretaceous Subcommission (Premoli Silva, 2010).

Following documentation of a major planktonic foraminiferal turnover across the Aptian-Albian interval (Huber and Leckie, 2011; Huber et al., 2011), the first occurrence of planktonic foraminiferan Microhedbergella renilaevs Huber and Leckie, 2011 was proposed as the biomarker to define the base of the Albian, first by Petrizzo et al. (2012, 2013) and then by Kennedy et al. (2014).

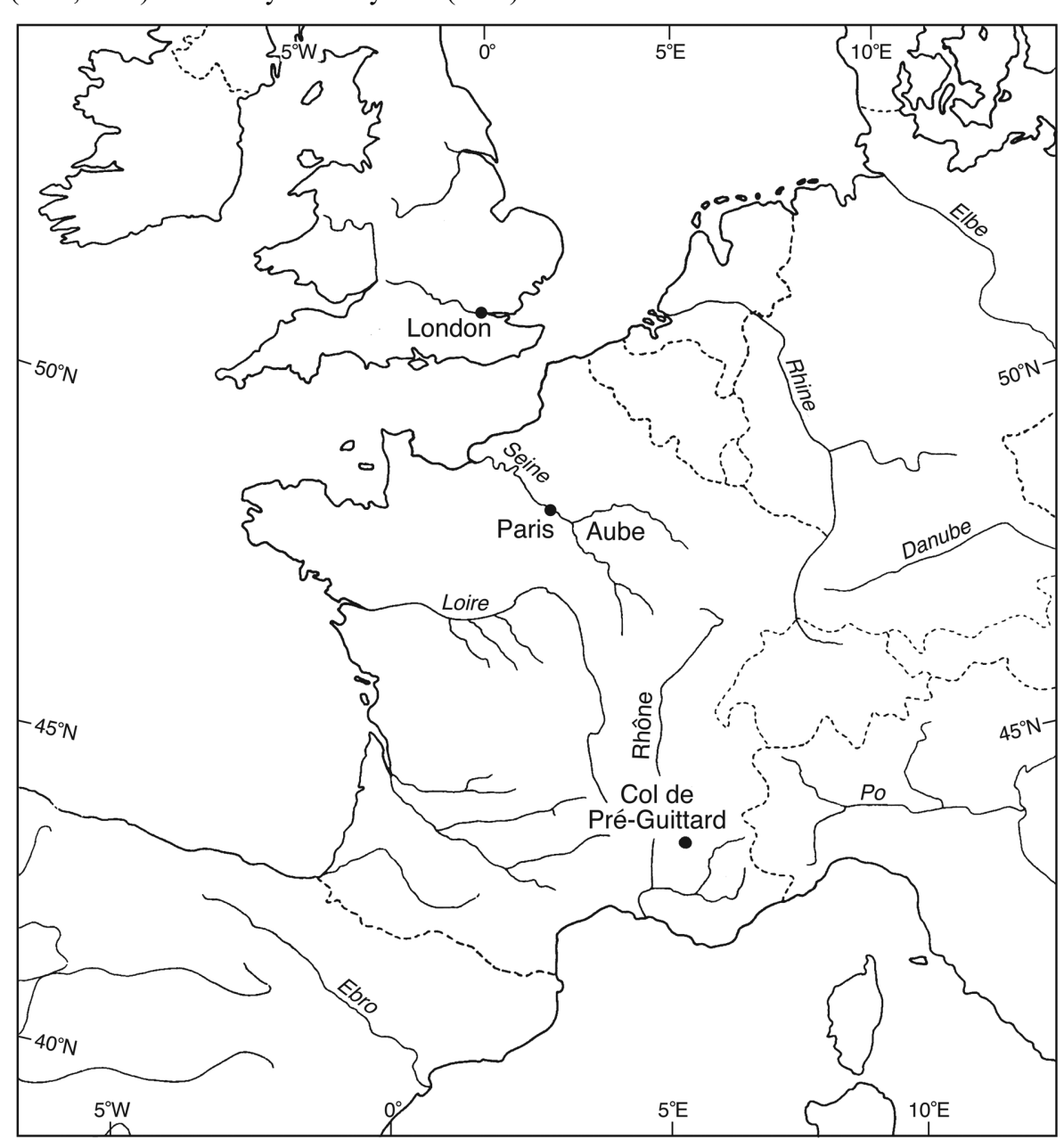

Figure 1. Localities in France mentioned in the text.

\section{The Global Boundary Stratotype Section and Point for the Base of the Albian Stage}

\section{Location}

The GSSP lies north of the 877 spot height east of the D173 road, $400 \mathrm{~m}$ south of the Ferme de Pré-Guittard, and $830 \mathrm{~m}$ SSE of the Col de Pré-Guittard in the Commune of Arnayon (Figs. 1-3). The PréGuittard section (Figs. 1, 2, and 5) lies $11 \mathrm{~km}$ north-northwest of Rémuzat and $19 \mathrm{~km}$ northwest of Rosans in the Départment of Drôme at $44^{\circ} 29^{\prime} 47^{\prime \prime} \mathrm{N}, 5^{\circ} 18^{\prime} 41^{\prime \prime} \mathrm{E}$ on the 125:000 topographic sheet Série Bleu 3138E, La Motte-Chalançon (It should be noted that although referred to as the Col de Pré-Guittard section in the literature it is some distance to the south of the col, as noted above).

\section{Access}

The GSSP is reached by taking the D173 west from its junction with the D61, $2 \mathrm{~km}$ south of La Motte-Chalançon. The locality lies above and below the road just north of spot height $877,400 \mathrm{~m}$ east of south of the Ferme Pré-Guittard. Previous key accounts of the section are presented by Bréhéret et al. (1986), Bréhéret (1997 and references therein), Kennedy et al. $(2000,2014)$ and Petrizzo et al. $(2012,2013)$.
Description of the Global Stratotype Section

The section is exposed over a distance of several hundred metres in a series of gullies and ravines in outcrops on the eastern and western sides of the D173 road (Fig. 2). A general view of the outcrop to the east is shown in Figure 3, and a lithostratigraphic log in Figure 4. As the name suggests, the Marnes Bleues Formation is a predominantly argillaceous sequence with a varying but generally low carbonate fraction. The base is drawn at the boundary with the highest well-cemented limestone of the Faisceau Fromaget (Figs. 3 and 4), which provides the zero datum in this account. There is a series of marker beds of regional extent:

- The Niveau Jacob at the 2.5 to $4.0 \mathrm{~m}$ level. This is an interval with laminae rich in organic matter with bedding planes covered in crushed ammonites.

- The Niveau Kilian the base of which is at the 37-m level; a meter-thick unit moderately rich in organic matter, with some laminated intervals.

- The Niveau Paquier, the base of which is at the 68-m level; a 1.5-m unit of black, laminated organic-rich shale. Individual bedding planes are plastered with ammonites with powdery remnants of original white aragonitic shell material. 


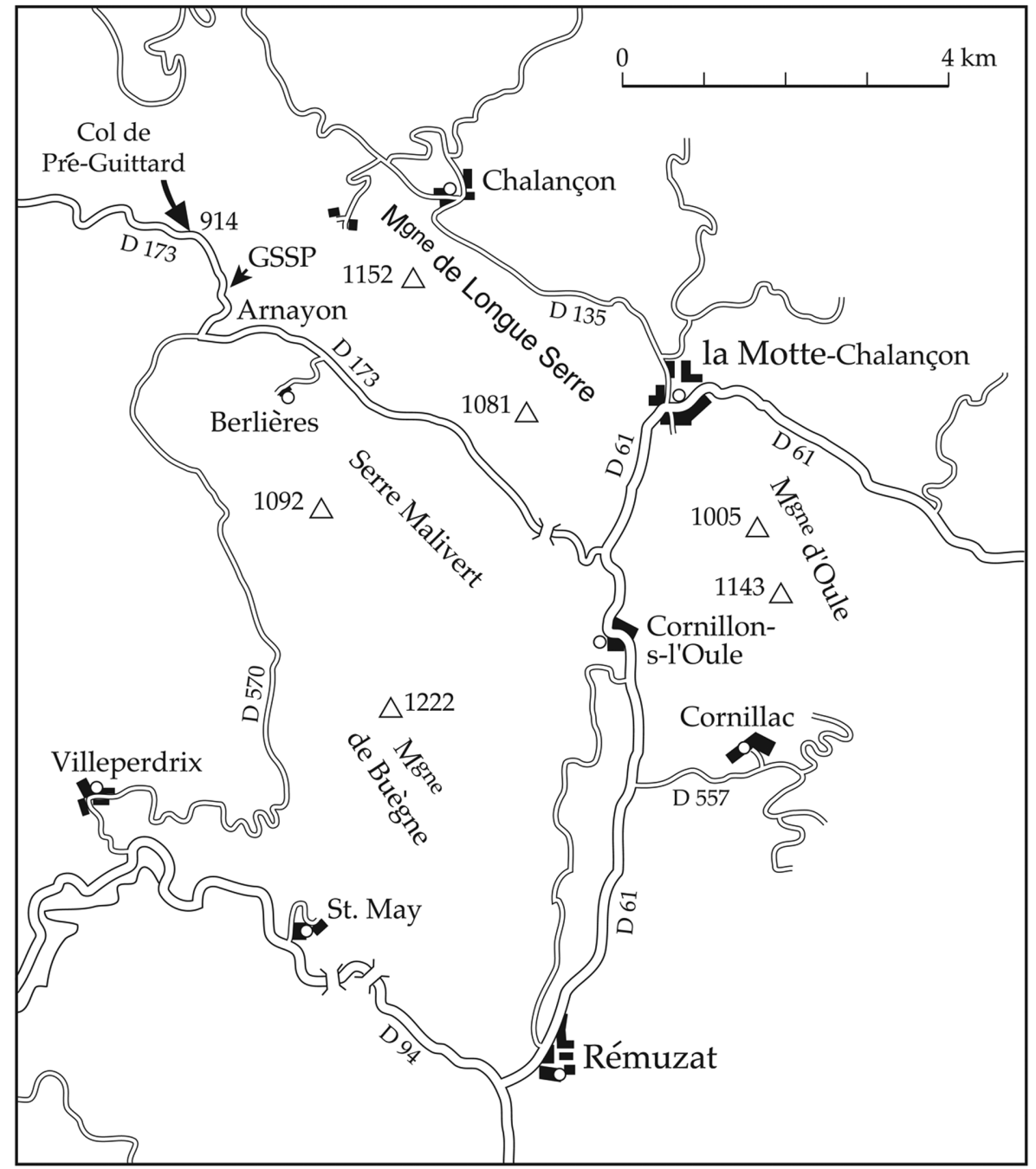

Figure 2. Location map for the Col de Pré-Guittard, Arnayon (Drôme). Rémuzat lies about $50 \mathrm{~km}$ northeast of Orange.

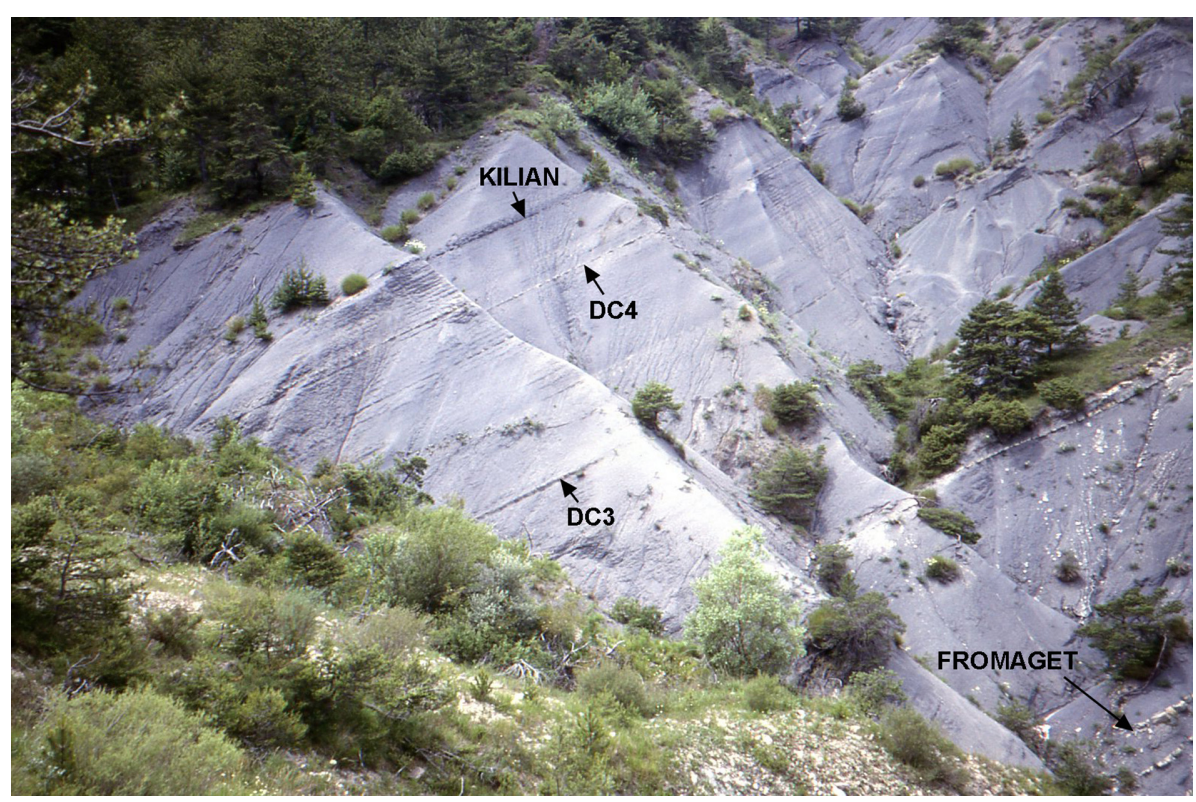

Figure 3. The Global boundary Stratotype Section: view from the D173 road. DC = "délits calcaires" of Bréhérét (1997).
- The Niveau Leenhardt, a pair of laminated organic-rich shales with fish debris, ammonites, and inoceramid bivalves, the base being at the $101.5-\mathrm{m}$ level.

Also present are a series of what Bréhérét (1997, p. 9) referred to as "délits calcaires (calcaires délités)" (DC) of which there are five in the lower $45 \mathrm{~m}$ of the section (Figs. 3 and 4). As better cemented levels, their weathering profile makes them valuable secondary lithological markers in the sequence.

The Boundary Level: Primary and Auxiliary Markers

(1) The topmost limestone of the Faisceau Fromaget, PGO, the zero datum.

(2) The Niveau Jacob, at 2.5-4.0 m.

(3) The first occurrence of subcircular examples of the nannofossil Prediscosphaera columnata at $6 \mathrm{~m}$. [The columnata lineage is widely distributed in both Boreal and Tethyan Realms, Ocean Drilling Program (ODP), Deep Sea Drilling Project (DSDP), and International Ocean Discovery Program (IPOD)sites, and is used as a global marker.]

(4) The first occurrence of circular examples of the nannofossil Prediscosphaera columnata and the lowest occurrence of the nannofossil Helicolithus trabeculatus at $29.5 \mathrm{~m}$. (The columnata lineage is used as a global marker. H. trabeculatus is widely distributed in both Boreal and Tethyan realms, DSDP, ODP and IPOD sites.)

(5) The last occurrence of the planktonic foraminiferan Hedbergella infracretacea at $33.5 \mathrm{~m}$. (This species is cosmopolitan in marine settings beyond the inner shelf in all biogeographic realms.)

(6) The last occurrence of the planktonic foraminiferan Hedbergella aptiana at $34.75 \mathrm{~m}$. (This species is cosmopolitan in marine settings at middle shelf and greater depths with occurrences in all biogeographic realms.)

(7) The last occurrence of the planktonic foraminiferan Paraticinella rohri (= Ticinella bejaouensis and Paraticinella eubejaouensis in previous literature; see Premoli Silva et al., 2009; Ando et al., 2014) at 34.75 m. (This species is cosmopolitan in middle shelf and deeper marine settings with occurrences in all biogeographic realms, and is cosmopolitan in middle shelf and deeper marine settings.)

(8) The first occurrence of the planktonic foraminiferan Microhedbergella miniglobularis at $35 \mathrm{~m}$. [This species has been identified at northern subtropical deep sea sites on the Blake Plateau 
Col de Pré-Guittard

Vocontian Basin

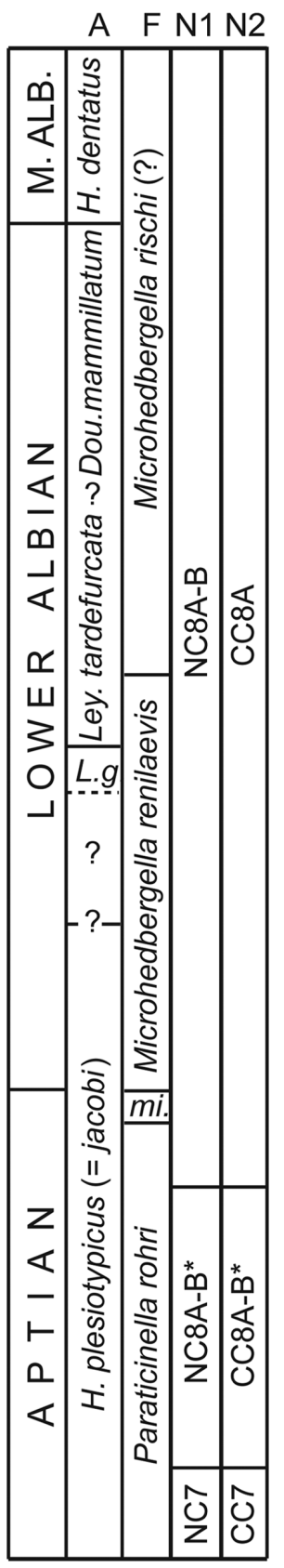

20

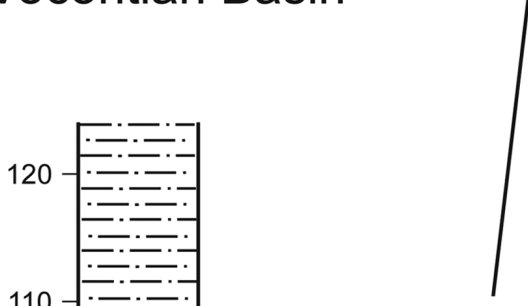

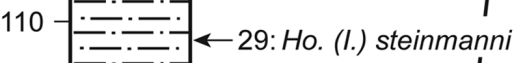

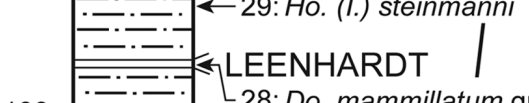

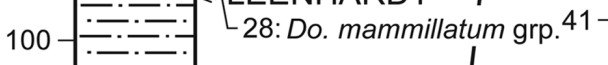
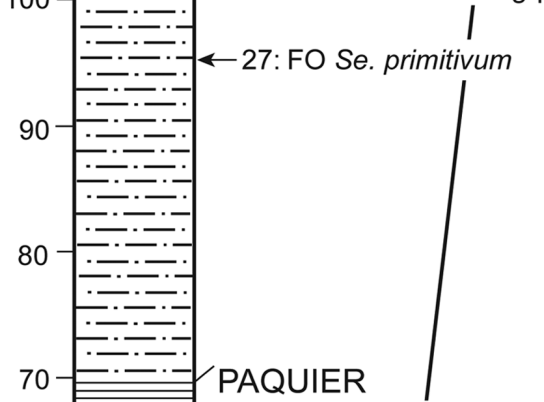

40

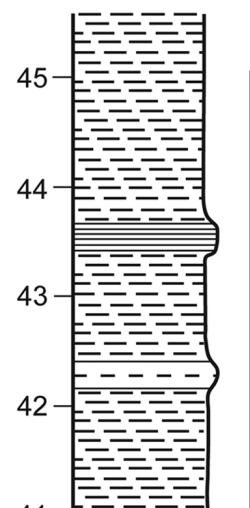

F N1 N2

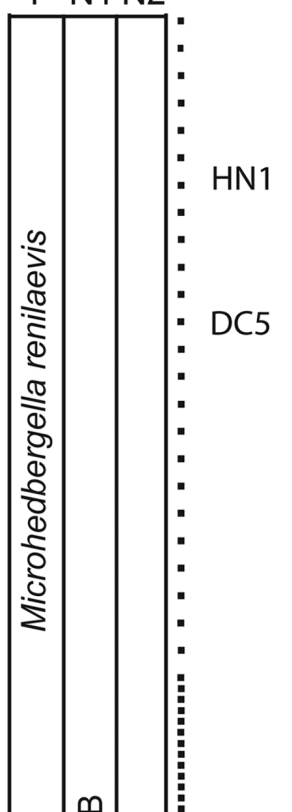

38

39

KILIAN

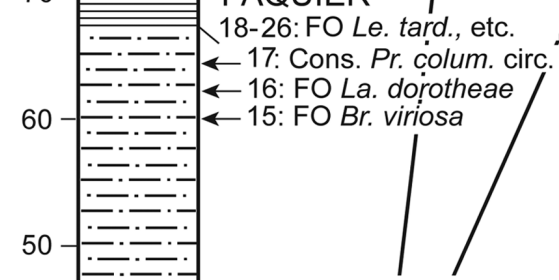

7

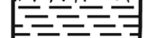

36

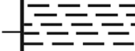

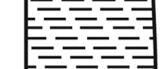

35

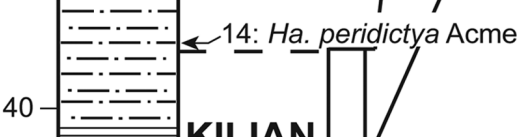

34
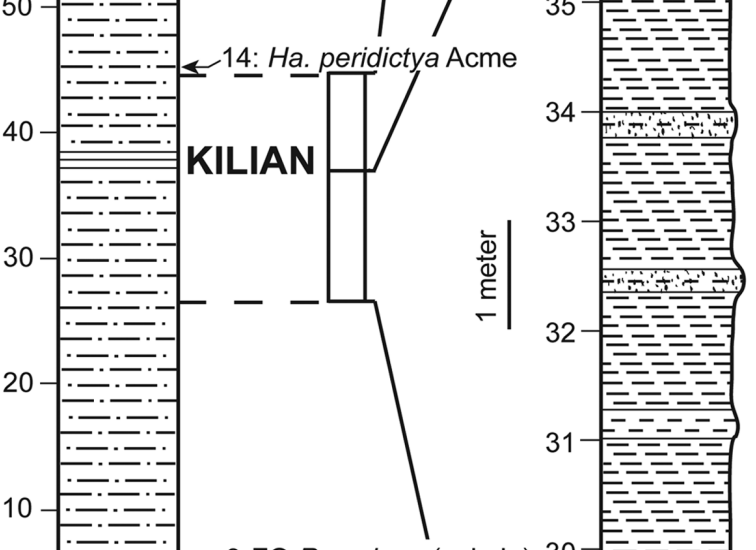

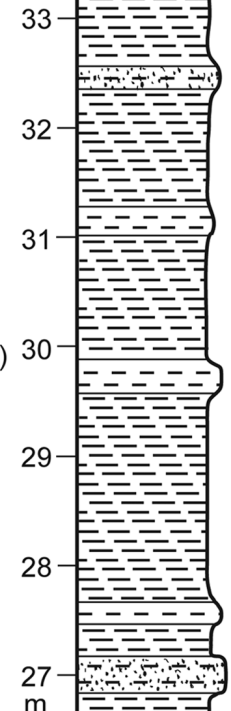

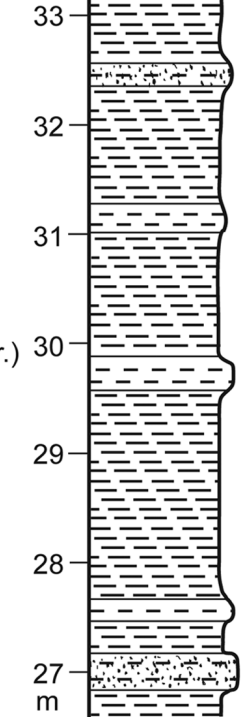

$\delta^{13} \mathrm{C}$ minimum;

$\rightarrow$ 12,13: FO Mi. renilaevis :10: LO Ps. blakenosensis -9: FO Ga. stenostaurion

- $\leftarrow$ 8: FO Mi. miniglobularis

- 6 6, 7: LO Hd. aptiana,

$-\longleftarrow 5$ : LO Hd. infracretacea

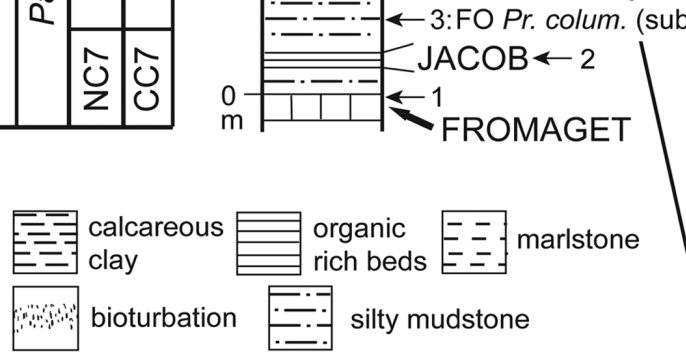

Figure 4. The succession at the Col de Pré-Guittard, Arnayon (Drôme), showing local marker beds Fromaget, Jacob, Kilian, Paquier and Leenhardt. Numbers 1-29 refer to the sequence of events described in the text for the GSSP including the first occurrence of the planktonic foraminiferan Microhedbergella renilaevis, event 13, at the $37.4 \mathrm{~m}$ level (modified after Petrizzo et al., 2012). See text for complete spellings and explanation for unnamed events. Columns include A (ammonites - Kennedy et al., 2000), PF (planktonic foraminifera - Petrizzo et al., 2013, with modification of Pa. rohri Zone, the equivalent of previously identified Pa. eubejaouaensis Zone), N1, calcareous nannofossils (NC = Roth 1978 scheme) and N2, calcareous nannofossils $(C C=$ Sissingh 1977 scheme $)$. FO = first occurrence, LO = last occurrence. DC = “délits calcaires” of Bréhérét (1997). Abbreviated ammonite names are; L.g., Leymeriella germanica; Ley, Leymeriella; Dou., Douvilleiceras; H., Hoplites. The small squares to the right of the right column represent levels of samples taken for the study of Petrizzo et al. (2012). 


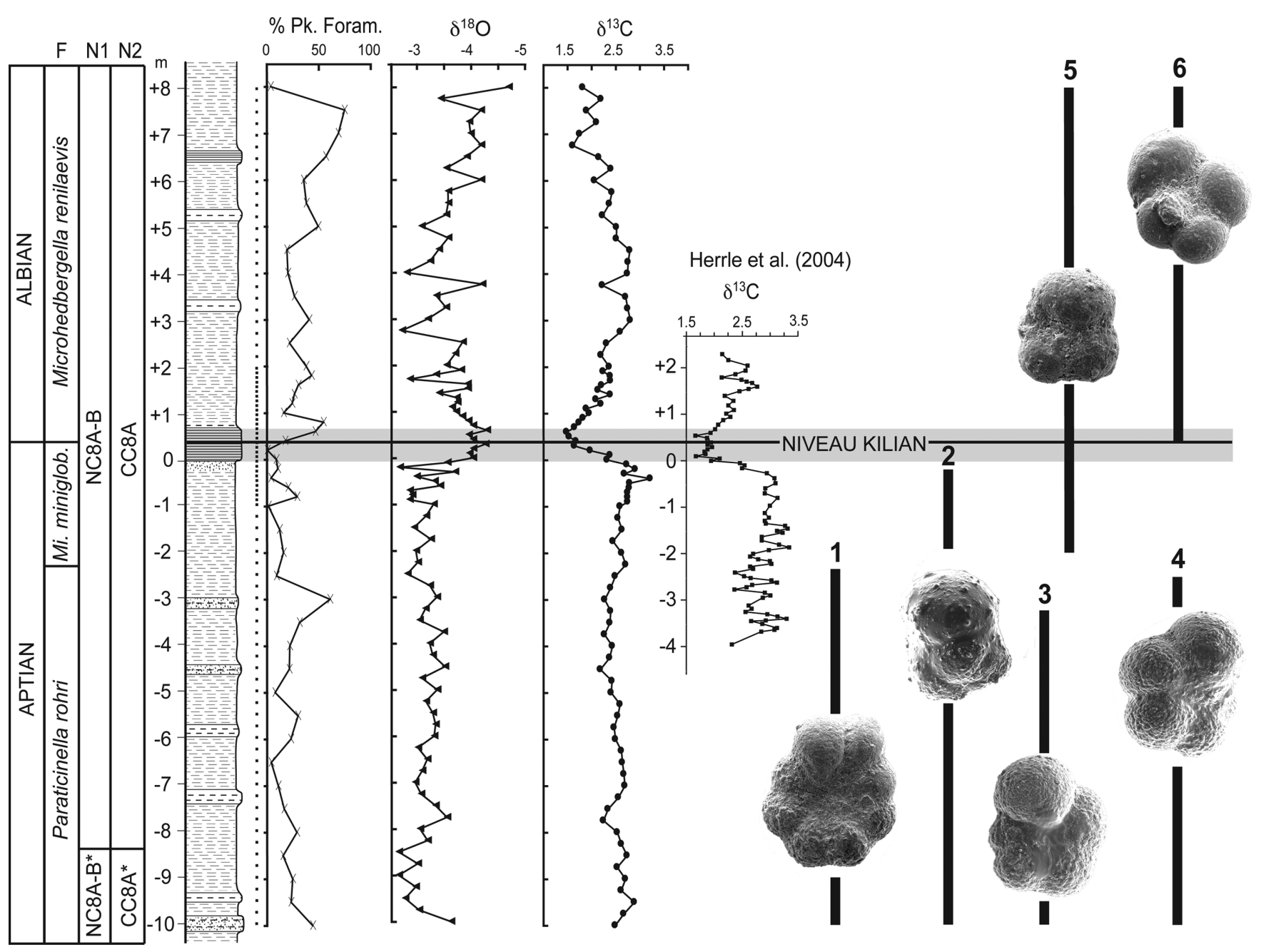

Figure 5. Abundance (\%) of planktonic foraminifera and oxygen- and carbon-stable isotope stratigraphy from Petrizzo et al. (2012, 2013), and carbon-isotope data from Herrle et al. (2004) through the Niveau Kilian at Pré-Guittard. Species illustrated (not to scale) with their ranges include: 1, Paraticinella rohri; 2, Pseudoguembelitria blakenosensis; 3, Hedbergella infracretacea; 4, Hedbergella aptiana; 5, Microhedbergella miniglobularis; 6, Microhedbergella renilaevis.

(North Atlantic, DSDP Site 390, ODP Site 1049) on the Falkland Plateau at southern high latitudes (southern South Atlantic, DSDP Site 511), the Exmouth Plateau (subtropical south-eastern Indian Ocean, Hole 763B) and in the western Tethys including the Vocontian Basin].

(9) The first occurrence of the nannofossil Gartnerago stenostaurion at $36 \mathrm{~m}$. (The species is widely distributed in both Boreal and Tethyan realms, DSDP, ODP and IPOD sites.)

(10) The last occurrence of the planktonic foraminiferan Pseudoguembelitria blakenosensis at $36.8 \mathrm{~m}$. (This species was recorded at Blake Nose ODP Holes 1049A, 1049B, and 1049C in the subtropical western North Atlantic and western Tethys, including the Vocontian Basin, southeast France.)

(11) The base of the laminated Niveau Kilian at $37 \mathrm{~m}$.

(12) The minimum value of the negative excursion of $\delta^{13} \mathrm{C}$ at 37.4 $\mathrm{m}$. (This is a global phenomenon.)

(13) The proposed candidate boundary marker: the first occurrence of the planktonic foramininferan Microhedbergella renilaevis at 37.4 $\mathrm{m}$. (This species has been identified at northern subtropical deep sea sites on the Blake Plateau (North Atlantic, DSDP Sites 390, ODP Site
1049) on the Falkland Plateau at southern, high latitudes (southern South Atlantic, DSDP Site 511), the Exmouth Plateau (subtropical southeastern Indian Ocean, Hole 763B) and in the western Tethys (Vocontian Basin, southeast France).

(14) The acme of the palynomorph Hapsocysta peridictya at $46 \mathrm{~m}$. (This palynomorph has a cosmopolitan distribution in ODP cores and occurs onshore in both Boreal and Tethyan realms.)

(15) The first occurrence of the nannofossil Broinsonia viriosa at $60 \mathrm{~m}$. (Originally described from the Boreal Realm (southern England), and now recorded from the Vocontian Basin)

(16) The first occurrence of the nannofossil Laguncula dorotheae, at $63.3 \mathrm{~m}$. (This species is widely distributed in both Boreal and Tethyan realms, DSDP, ODP and IPOD sites.)

(17) The first consistent occurrence of circular examples of the nannofossil Prediscosphaera columnata, at $66.6 \mathrm{~m}$. (This species is widely distributed in both Boreal and Tethyan realms, DSDP, ODP and IODP sites.)

(18) The base of the Niveau Paquier at $68 \mathrm{~m}$. This level coincides with a minor discontinuity at the Col de Pré-Guittard. 

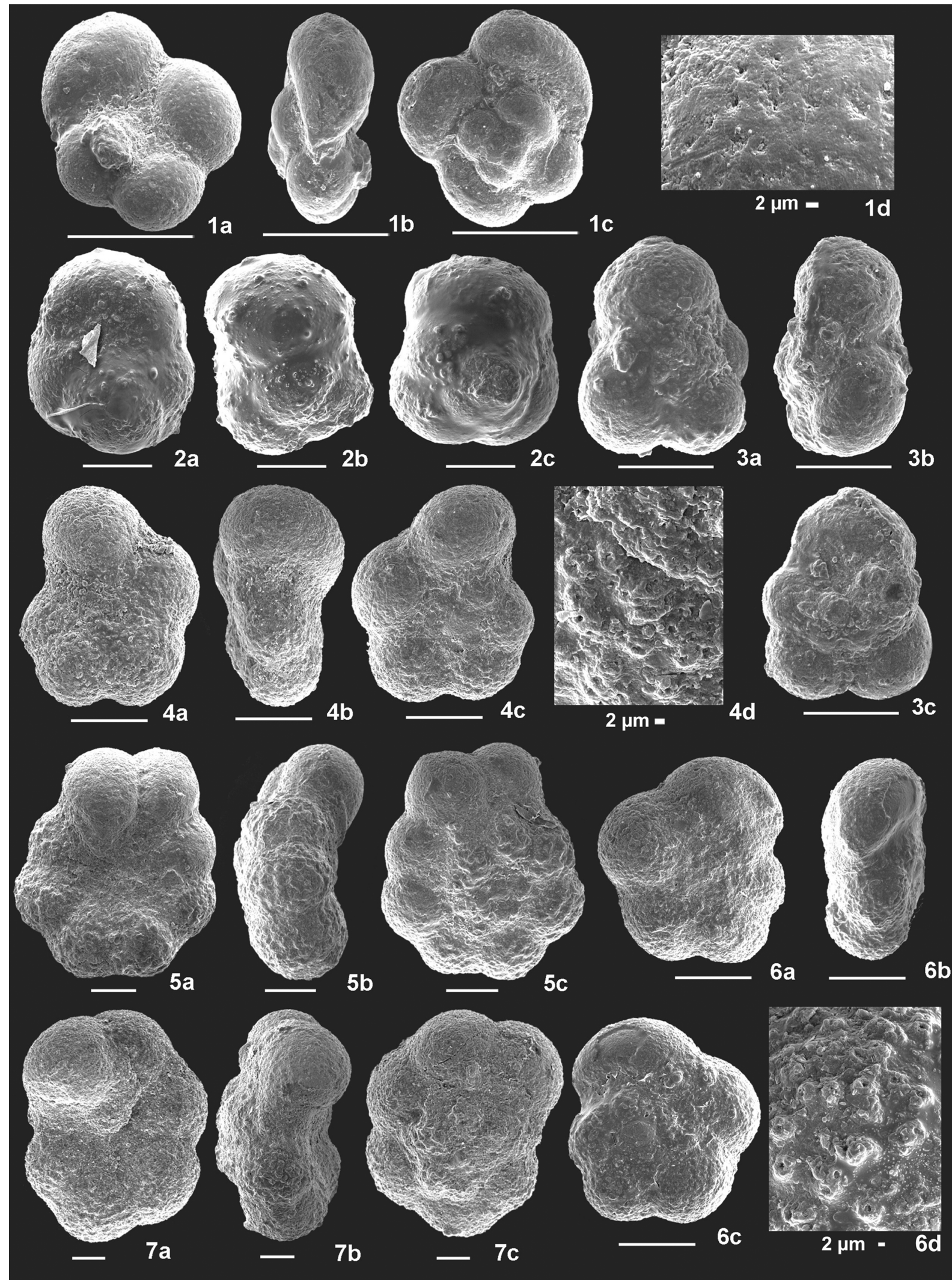

Figure 6. Planktonic foraminifera across the Niveau Kilian at Pré-Guittard illustrated in Petrizzo et al. (2012): (1a-c) Microhedbergella renilaevis, sample FK+7.0; (2a-c) Pseudoguembelitria blakenosensis, sample FK-0.8; (3a-c) Pseudoguembelitria blakenosensis, sample FK-9.0; Hedbergella aptiana, sample FK-9.0; (4a-d) Hedbergella aptiana, sample FK-9.0; (5a-c) Paraciticinella rohri, sample FK-9.0; (6a-d) Hedbergella infracretacea, sample FK-10.5; (7a-c) Paraticinella transitoria, sample-10.0. Scale bars represent 100 $\mu$ m for Figures 1 and 4-7, except where indicated otherwise, and $50 \mu m$ for Figures 2 and 3; $a=$ umbilical view, $b=$ lateral view, $c=$ spiral view. 


\begin{tabular}{|c|c|c|c|c|c|c|c|c|c|c|c|c|c|c|c|c|c|c|c|c|}
\hline $\begin{array}{l}\text { Col de } \\
\text { Pré- } \\
\text { Guittard } \\
\text { samples }\end{array}$ & $\frac{\infty}{\frac{\omega}{\omega}}$ & 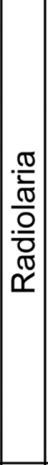 & 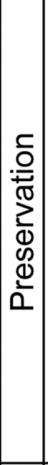 & 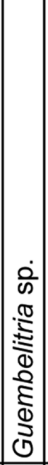 & 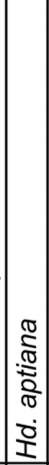 & 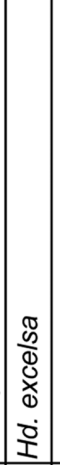 & 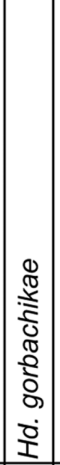 & 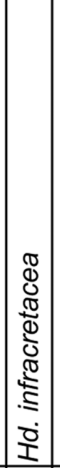 & 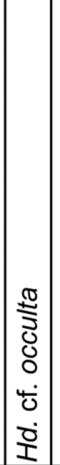 & 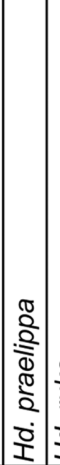 & 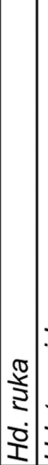 & 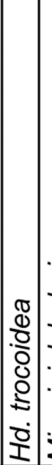 & 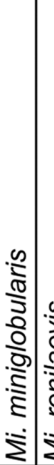 & 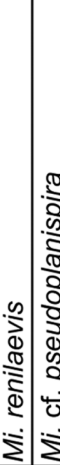 & 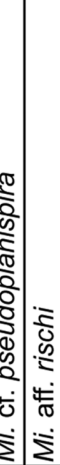 & 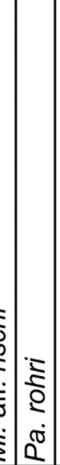 & 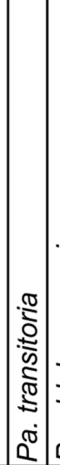 & 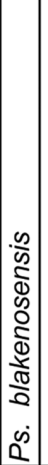 & $\stackrel{0}{\mathbb{2}}$ & 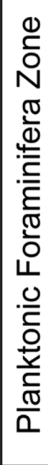 \\
\hline $\mathrm{FK}+8.0$ & 8.00 & & $M$ & & & & & & & & & & \begin{tabular}{l|l}
$x$ & $>$ \\
\end{tabular} & $\mathrm{x}$ & $x$ & 人 & & & \multirow{21}{*}{ 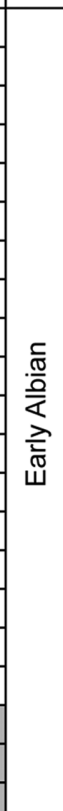 } & \multirow{20}{*}{$\begin{array}{l}0 \\
\vdots \\
\vdots \\
\vdots \\
\vdots \\
\vdots \\
\vdots \\
\vdots \\
\vdots \\
\vdots\end{array}$} \\
\hline $\mathrm{FK}+7.5$ & 7.50 & $R$ & $M$ & & & & & & & & & & \begin{tabular}{l|l}
$x$ & 2 \\
\end{tabular} & $\mathrm{x}$ & $x$ & 人 & & & & \\
\hline $\mathrm{FK}+7.0$ & 7.00 & & $\mathrm{M}$ & & & & & & & & & & \begin{tabular}{l|l}
$x$ & 2 \\
\end{tabular} & $\mathrm{x}$ & $x$ & र & & & & \\
\hline $\mathrm{FK}+6.5$ & 6.50 & & $M$ & & & & & & & & & & $x / 2$ & $x$ & $x$ & 安 & & & & \\
\hline $\mathrm{FK}+6.0$ & 6.00 & & $M$ & & & & & & & & & & \begin{tabular}{l|l}
$x$ & $\lambda$ \\
\end{tabular} & $\mathrm{X}$ & $x$ & र & & & & \\
\hline $\mathrm{FK}+5.5$ & 5.50 & & $M$ & & & & & & & & & & \begin{tabular}{l|l}
$x$ & $>$ \\
\end{tabular} & $\mathrm{x}$ & $x$ & र & & & & \\
\hline $\mathrm{FK}+5.0$ & 5.00 & $R$ & $\mathrm{P}$ & & & & & & & & & & \begin{tabular}{l|l}
$x$ & 1 \\
\end{tabular} & $\mathrm{x}$ & $x$ & K & & & & \\
\hline $\mathrm{FK}+4.5$ & 4.50 & & $\mathrm{P}$ & & & & & & & & & & \begin{tabular}{l|l}
$x$ & $>$
\end{tabular} & $x$ & & & & & & \\
\hline $\mathrm{FK}+4.0$ & 4.00 & $R$ & $M$ & & & & & & & & & & $\begin{array}{ll}\mathrm{X} & \mathrm{\gamma} \\
\end{array}$ & $x$ & & & & & & \\
\hline $\mathrm{FK}+3.5$ & 3.50 & & $\mathrm{P}$ & & & & & & & & & & \begin{tabular}{l|l}
$x$ & $>$ \\
\end{tabular} & $\mathrm{x}$ & & & & & & \\
\hline $\mathrm{FK}+3.0$ & 3.00 & & $\mathrm{P}$ & & & & & & & & & & $\mathrm{X}>$ & $x$ & & & & & & \\
\hline$\overline{F K}+2.5$ & 2.50 & & $\mathrm{P}$ & & & & & & & & & & \begin{tabular}{l|l}
$x$ & $>$
\end{tabular} & $\mathrm{x}$ & & & & & & \\
\hline $\mathrm{FK}+2.0$ & 2.00 & & $\mathrm{P}$ & & & & & & & & & & \begin{tabular}{l|l}
$x$ & $>$ \\
\end{tabular} & $\mathrm{x}$ & & & & & & \\
\hline $\mathrm{FK}+1.8$ & 1.80 & & $\mathrm{P}$ & $X ?$ & & & & & & & & & \begin{tabular}{l|l}
$x$ & 1 \\
\end{tabular} & $\mathrm{x}$ & & & & & & \\
\hline $\mathrm{FK}+1.6$ & 1.60 & $R$ & $\mathrm{P}$ & $\mathrm{X}$ & & & & & & & & & 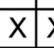 & $x$ & $x$ & र & & & & \\
\hline $\mathrm{FK}+1.4$ & 1.40 & & $\mathrm{P}$ & $X ?$ & & & & & & & & & \begin{tabular}{l|l}
$x$ & 1 \\
\end{tabular} & $\mathrm{x}$ & $x$ & र & & & & \\
\hline $\mathrm{FK}+1.2$ & 1.20 & $R$ & $M$ & & & & & & & & & & \begin{tabular}{l|l}
$x$ & $\lambda$ \\
\end{tabular} & $\mathrm{x}$ & - & & & & & \\
\hline$\overline{F K}+1.0$ & 1.00 & $R$ & $\mathrm{P}$ & & & & & & & & & & \begin{tabular}{l|l}
$x$ & $\lambda$ \\
\end{tabular} & $\mathrm{x}$ & $x$ & र & & & & \\
\hline $\mathrm{FK}+0.8$ & 0.80 & & $\mathrm{P}$ & & & & & & & & & & \begin{tabular}{l|l}
$x$ & 2 \\
\end{tabular} & $\mathrm{x}$ & & & & & & \\
\hline $\mathrm{FK}+0.6$ & 0.60 & & $M$ & & & & & & & & & & \begin{tabular}{l|l}
$x$ & $>$
\end{tabular} & \begin{tabular}{l|l}
$x$ & $x$ \\
\end{tabular} & \begin{tabular}{l|l}
$x$ & $x$
\end{tabular} & र & & & & \\
\hline $\mathrm{FK}+0.4$ & 0.40 & & $\mathrm{P}$ & & & $\mathrm{X}$ ? & & & & & & & \begin{tabular}{l|l}
$x$ & 1 \\
\end{tabular} & $\mathrm{x}$ & & & & & & \\
\hline$\overline{F K}+0.2$ & \begin{tabular}{l|l}
0.20 \\
\end{tabular} & & $\mathrm{P}$ & & & & & & & & & & & & & & & & \multirow{27}{*}{$\frac{5}{2}$} & \multirow{27}{*}{ 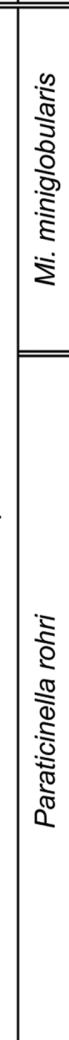 } \\
\hline FK 0 & 0.00 & & $P$ & & & & & & & & & & $x$ & & $x$ & र & & & & \\
\hline FK -0.2 & -0.20 & $\mathrm{R}$ & $\mathrm{P}$ & & & & & & & & & & $x$ & & $x$ & र & & $\mathrm{x}$ & & \\
\hline FK - 0.4 & \begin{tabular}{l|}
-0.40 \\
\end{tabular} & & $\mathrm{P}$ & & & & & & $\mathrm{X}$ & & & & $\mathrm{x}$ & & \begin{tabular}{l|l}
$x$ & $x$ \\
\end{tabular} & K & & & & \\
\hline FK -0.6 & -0.60 & $R$ & $\mathrm{P}$ & & & & & & & & & & $\mathrm{x}$ & $x$ & \begin{tabular}{l|l}
$x$ & $x$ \\
\end{tabular} & K & & & & \\
\hline FK -0.8 & \begin{tabular}{l|l|}
-0.80 \\
\end{tabular} & $R$ & $M$ & & & $x$ & & & & & & & $x$ & & & & & $x$ & & \\
\hline FK -1.0 & \begin{tabular}{l|l|}
-1.00 \\
\end{tabular} & $\mathrm{R}$ & $\mathrm{P}$ & & & & & & & & & & $\mathrm{x}$ & & & & & & & \\
\hline FK -1.5 & -1.50 & & $\mathrm{P}$ & & & $\mathrm{X}$ & & & $\mathrm{X}$ & & & & & & $x$ & र & & & & \\
\hline FK -2.0 & -2.00 & & $\mathrm{P}$ & & & & & & & $\mathrm{X}$ ? & & & $\mathrm{X}$ & & $x$ & & $\mathrm{X}$ & $\mathrm{x}$ & & \\
\hline$\overline{F K}-2.25$ & \begin{tabular}{l|l}
-2.25 \\
\end{tabular} & & $\mathrm{PP}$ & & $\mathrm{X}$ & & & & & & & & & & & $\mathrm{X}$ & & & & \\
\hline FK -2.5 & -2.50 & & $\mathrm{P}$ & & & & & & & & & & & & $\mathrm{X}$ & \begin{tabular}{|l|l|} 
& $x$ \\
\end{tabular} & & $\mathrm{x}$ & & \\
\hline FK -3.0 & -3.00 & & $\mathrm{P}$ & & $\mathrm{X}$ & & & & & & & & & & $x$ & $x$ & & $\mathrm{x}$ & & \\
\hline FK -3.5 & -3.50 & & $\mathrm{P}$ & & $X$ & & & $X ?$ & & & & & & & $x$ & \begin{tabular}{|l|l|}
$x$ \\
\end{tabular} & & & & \\
\hline FK -4.0 & $\begin{array}{l}-4.00 \\
\end{array}$ & & $M$ & & $\mathrm{X}$ & & & $x ?$ & & & & & & & $x$ & k & & $x$ & & \\
\hline FK -4.25 & $\begin{array}{l}-4.25 \\
\end{array}$ & & $\mathrm{P}$ & & $x$ & & & $x$ & & & & & & & & $x$ & & & & \\
\hline FK -4.5 & -4.50 & $R$ & $\mathrm{P}$ & & $\mathrm{X}$ & & & $x$ & & & & & & & $x$ & $x$ & $x$ & & & \\
\hline FK -5.0 & -5.00 & & $\mathrm{P}$ & & $\mathrm{X}$ & & & & & & & & & & & & & $\mathrm{x}$ & & \\
\hline FK -5.5 & $\begin{array}{l}-5.50 \\
\end{array}$ & & \begin{tabular}{|l|}
$M$ \\
\end{tabular} & & & & & $x$ & & & & & & & & $\mathrm{X}$ & & $x$ & & \\
\hline FK -6.0 & -6.00 & & $\mathrm{P}$ & & $\mathrm{X}$ & & & & & & $\mathrm{x}$ & & & & & $\mathrm{X}$ & & $x$ & & \\
\hline FK -6.5 & \begin{tabular}{l|}
-6.50 \\
\end{tabular} & & $\mathrm{P}$ & & $X$ & & & & & & & & & & & & & & & \\
\hline FK -7.0 & -7.00 & & $\mathrm{P}$ & & & & & & & & & & & & & $\mathrm{X}$ & $\mathrm{x}$ & $x$ & & \\
\hline FK -7.5 & -7.50 & $\mathrm{R}$ & $\mathrm{P}$ & & $\mathrm{X}$ & & & & & $\mathrm{X}$ & $\mathrm{x}$ & & & & & $\mathrm{X}$ & $\mathrm{x}$ & & & \\
\hline FK -8.0 & -8.00 & $R$ & $M$ & & & & & & & $\mathrm{x}$ & & & & & & $\mathrm{X}$ & $x$ & $x$ & & \\
\hline FK -8.5 & -8.50 & & $M$ & & & & & & & $\mathrm{x}$ & $\mathrm{x}$ & $\mathrm{x}$ & & & & $\mathrm{X}$ & & $\mathrm{x}$ & & \\
\hline FK -9.0 & \begin{tabular}{|c|}
-9.00 \\
\end{tabular} & & $M$ & & $\mathrm{X}$ & & $x$ & $x$ & & $\mathrm{X}$ & $x$ & & & & & $\mathrm{X}$ & $\mathrm{x}$ & $x$ & & \\
\hline FK -9.5 & \begin{tabular}{|l|}
-9.50 \\
\end{tabular} & & $M$ & & $\mathrm{X}$ & $\mathrm{X}$ & $\mathrm{X}$ & $\mathrm{X}$ & & $\mathrm{X}$ & \begin{tabular}{|l|}
$x$ \\
\end{tabular} & & & & & $\mathrm{X}$ & $\mathrm{X}$ & $\mathrm{x}$ & & \\
\hline FK -10 & $\mid-10.00$ & & $\mathrm{M}$ & & $\mathrm{X}$ & $\mathrm{X}$ & $\mathrm{X}$ & $\mathrm{X}$ & & $\mathrm{X}$ & $\mathrm{X}$ & & & & & $\mathrm{X}$ & $x$ & $\mathrm{x}$ & & \\
\hline
\end{tabular}

Figure 7. Stratigraphic distribution of planktonic foraminifera in the Col de Pré-Guittard section. 

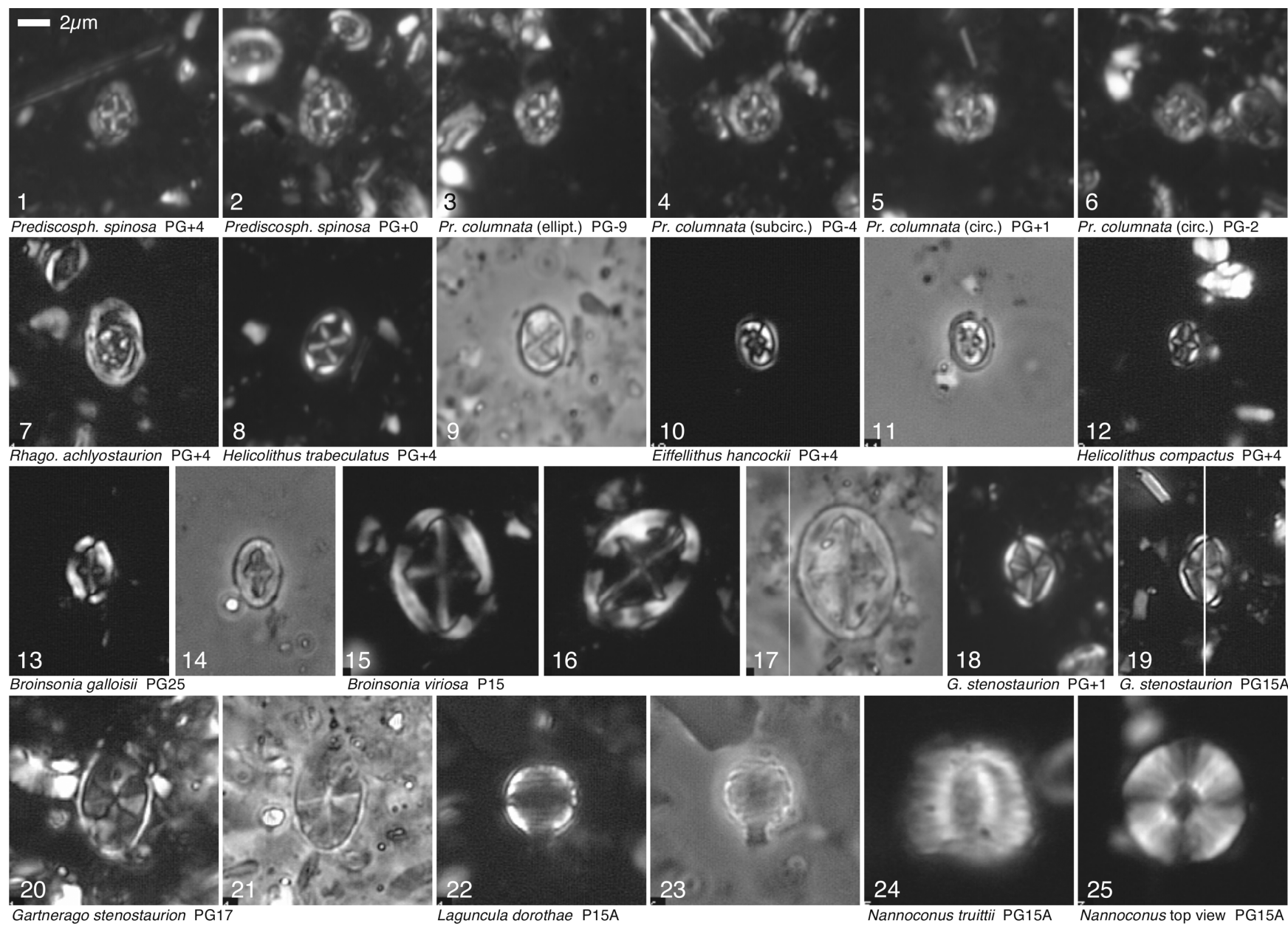

Figure 8. Nannofossils from the Col de Pré-Guittard section. 1, 2: Prediscospharea spinosa, 1: PG+4, 2: PG+0; 3: Prediscosphaera columnata (elliptical form), PG-9; 4, 5: Prediscosphera columnata (subcircular), 4: PG-4, 5: PG-1; 6: PG-2; 7: Rhagodiscus achyostaurion, PG-4; 8, 9: Helicolithus trabeculatus, PG+4; 10, 11: Eifellithus hancockii, PG+4; 12: Helicolithus compactus, PG+4; 13, 14: Broinsonia galloisi, PG25; 15-17: Broinsonia viriosa, PG15; 18-22, Gartnerago stenostaurion, 18: PG+1, 19: PG15A, 20, 21: PG17, 22; 23: Laguncula dorothae, PG15a; 24, 25: Nannoconus truittii.

(19) The first occurrence of the ammonite Leymeriella ( $L$ ) tardefurcata at the base of the Niveau Paquier at $68 \mathrm{~m}$. This datum corresponds to a distinctive geochemical signal in the organic matter present, a result of a significant contribution from Archaea (Kuypers et al., 2001, 2002). (The currently known distribution of Leymeriella (L) tardefurcata is Ardennes, Meuse, Aube, Drôme, Hautes Alpes, Alpes-deHaute-Provence and Isère in France, southern England, Denmark, Germany, Switzerland, Austria, Bulgaria, the Caucasus, Kopetdag, Iran, and Turkmenistan.) The first occurrence of Leymeriella tardefurcata is a useful proxy for the base of the Albian in successions without calcareous microfossils (Seyed-Emami and Wilmsen, 2016).

(20) The first occurrence of the bivalve Actinoceramus salomoni coptensis at the base of the Niveau Paquier at $68 \mathrm{~m}$. (The currently known distribution of the bivalve Actinoceramus salomoni coptensis is southern England, south-eastern France and Kazakhstan; salomoni sensu stricto is known from southern England, France, Switzerland, Kazkhstan, Georgia, and Azerbaijan. The Actinoceramus lineage is cosmopolitan.)

(21) The distinctive negative carbon-isotope excursion that begins just above the base of the Niveau Paquier in the Vocontian Basin, and is a local manifestation of Oceanic Anoxic Event (OAE) 1b. (This is a global event.)

(22) The first occurrence of the ammonite genus Douvilleiceras within the Niveau Paquier. (The currently known distribution of this genus is southern England, France, Switzerland, Germany, the Helvetic Zone of western Austria, Poland, Bulgaria, Romania, eastwards to Kazakhstan, Turkmenia, northern India, Pakistan, Japan, British Columbia, California, Arizona, New Mexico, and Texas in the United States, Peru, Colombia, Brazil, Tunisia, Algeria, Gabon, Angola, KwaZulu-Natal in South Africa, Mozambique, Somalia, and Madagascar.)

(23) The first occurrence of the ammonite genus Oxytropidoceras within the Niveau Paquier. (The geographic distribution of this genus extends from Western Europe (where it is very rare outside of southeastern France) to Morocco, Tunisia, Angola, KwaZulu-Natal in South Africa, Madagascar, Pakistan, California, Texas, Mexico, Puerto Rico, Venezuela, Colombia, Peru, and Brazil.)

(24) The last occurrence of the ammonite Hypacanthoplites anglicus in the upper part of the Niveau Paquier. (The currently known distribution of this species is southeast and northern France, southern England, Germany, the Caucasus and Central Asia.)

(25) The termination of the negative carbon-isotope excursion, 


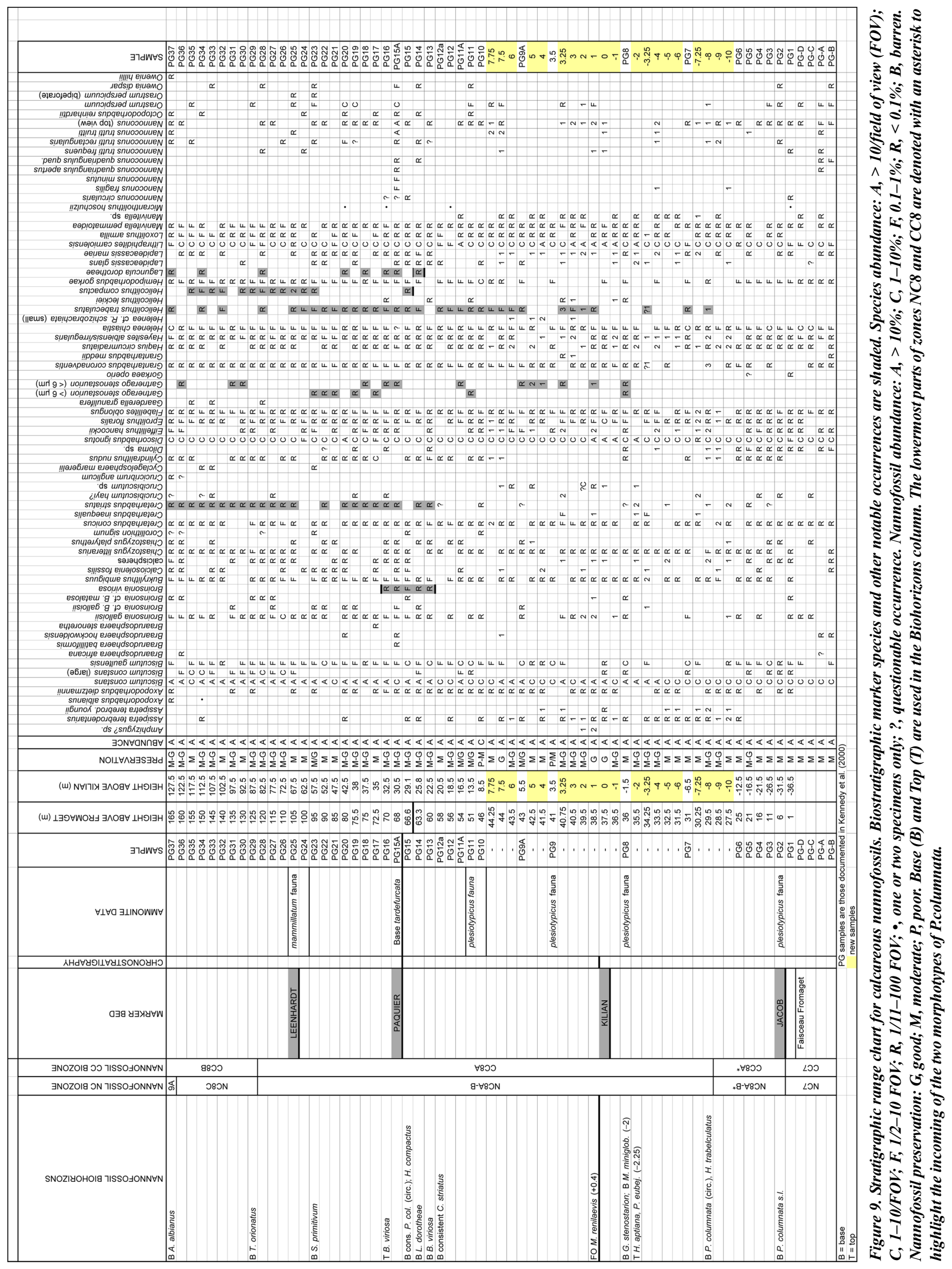




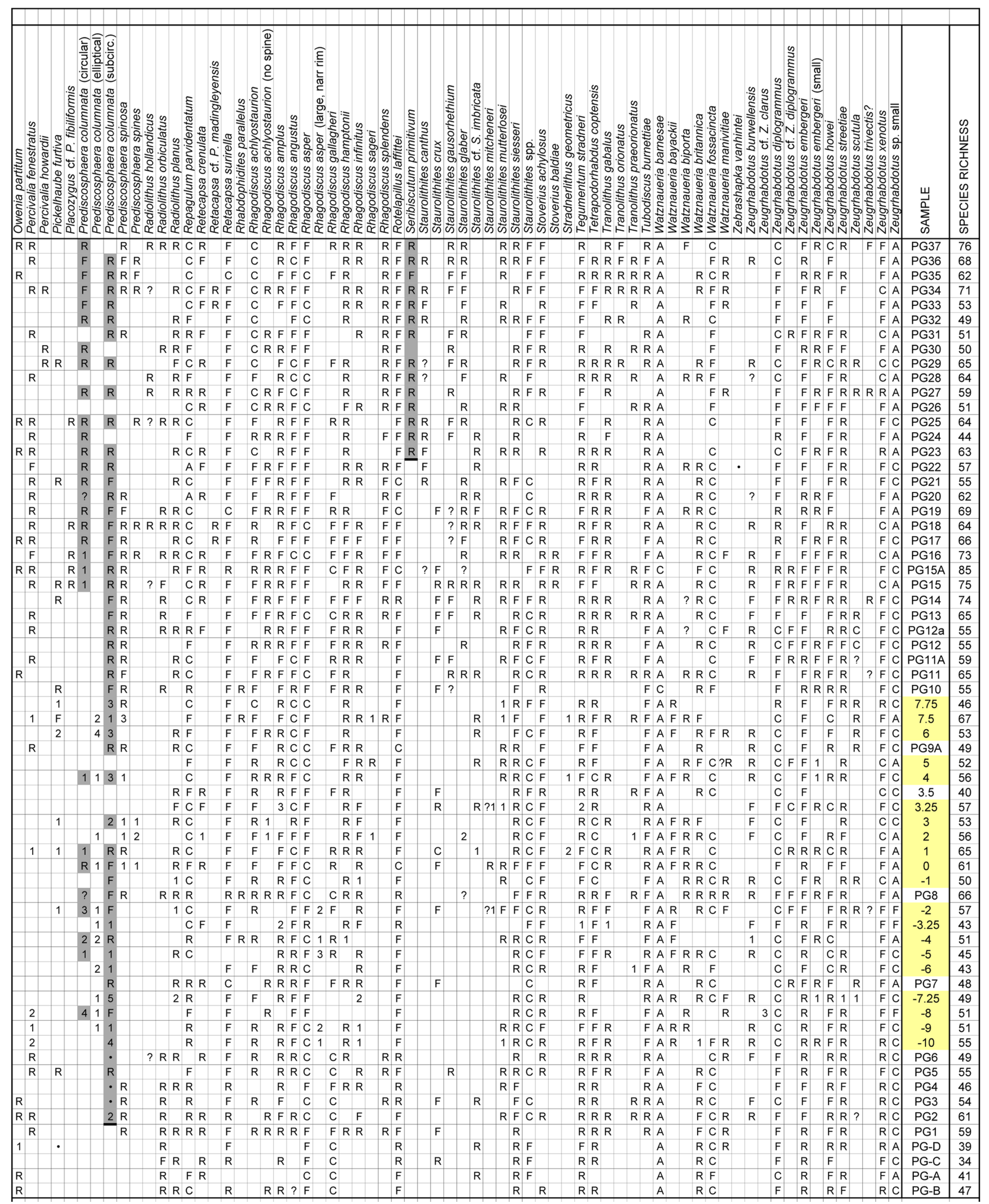

Figure 9. (continued).

which is situated at the top of the Niveau Paquier (OAE 1b) in the Col de Pré-Guittard section at approximately $70 \mathrm{~m}$. (This is a global event.)

(26) The last occurrence of the nannofossil Broinsonia viriosa at $70 \mathrm{~m}$.
(Originally described from Boreal Realm (southern England), and now recorded from the Vocontian Basin.)

(27) The first occurrence of the nannofossil Seribiscutum primi- 
tivum at $95 \mathrm{~m}$. (This species is widely distributed in both Boreal and Tethyan realms, DSDP, ODP and IODP sites.)

(28) The Niveau Leenhardt, with ammonites of the Douvilleiceras mammillatum group, $101.5 \mathrm{~m}$ above the top of the Faisceau Fromaget at Pré-Guittard. (The currently known distribution of ammonites of the Douvilleiceras mammillatum group is southern England, France, Germany, Switzerland, Poland, Romania, Bulgaria, Kazakhstan, Turkmenistan, Iran, north-western India, northern Pakistan, Tunisia, Angola, northern KwaZulu-Natal in South Africa, Madagascar, and possibly Peru.)

(29) The occurrence of the ammonite Hoplites (Isohoplites) steinmanni, $109.5 \mathrm{~m}$ above the top of the Faisceau Fromaget at Pré-Guittard. (The currently known distribution of this species is southern England, France, Germany, Switzerland, Austria, Poland, Bulgaria, Russia, and Kazakhstan.)

Figure 4 plots selected markers against a lithostratigraphic log of the section. Figure 5 plots stable isotope data and planktonic foraminiferal ranges. Figure 6 illustrated key planktonic foraminifera. Figure 7 plots the stratigraphic distribution of planktonic forminifera. Figure 8 illustrates key nannofossils. Figure 9 plots the stratigraphic distribution of nannofossils.

\section{Conclusions}

It will be seen that the Pré-Guittard section provides:

- A Global boundary Stratotype Section and Point for the base of the Albian Stage that can be identified using the first occurrence of the planktonic foraminiferan Microhedbergella renilaevis, set within a matrix of secondary markers.

- The boundary point lies within the widely recognized crisis interval that affected planktonic foraminifera over wide areas of the globe within the lowermost NC8/CC8 nannofossil Zone.

- The boundary point coincides with the minimum value of a negative excursion of approximately $1 \%$ in carbonate $\mathrm{d}^{13} \mathrm{C}$ that can be traced into the Atlantic region (Trabucho Alexandre et al., 2011).

- The boundary point lies some distance beneath the onset of the negative stable carbon-isotope excursion associated with the Niveau Pacquier, recording the globally recognizable OAE $1 \mathrm{~b}$, as demonstrated through the work of Herrle (2002; see also Herrle, 2003; Herrle and Mutterlose, 2003; Herrle et al., 2003; Herrle et al., 2004) elsewhere in the Vocontian Basin, and recognised in the Col de Pré-Guittard section. It should be noted that some authors include the Niveau Kilian as a partial manifestation of a longer lasting episodic OAE1b or OAE1b cluster to accompany the Niveau Paquier and Niveau Jacob (Leckie et al., 2002: Trabucho Alexandre et al., 2011).

- The succession that contains the boundary is rhythmically bedded in the Vocontian Basin, and so has the potential for development of an orbital timescale.

\section{Acknowledgements}

We thank Alessia Barchetta for her assistance during the earlier stages of this project, David Sansom (Oxford) for his assistance in drafting the figures for this report, and Markus Wilmsen (Dresden) for his review of the manuscript of this contribution. Figures 1, 2, and 4-9 are reproduced from Kennedy et al. (2014), published in Cretaceous Research, v. 51, pp. 248-259.

\section{References}

Amédro, F., Magniez-Jannin, F., Colleté, C., and Fricot, P., 1995, L'Albien - type de l'Aube, France une révision nécessaire: Géologie de la France, v. 2, pp. 25-42.

Ando, A., Huber, B.T., and Premoli Silva, I., 2014, Paraticinella rohri (Bolli, 1959) as the valid name for the latest Aptian zonal marker species of planktonic foraminifera traditionally called bejaouaensis or eubejaouaensis: Cretaceous Research, v. 45, pp. 275-287.

Bréhéret, J.-G., 1997, L'Aptien et l'Albien de la Fosse Vocontienne (des bordures au bassin). Evolution de la sédimentation et enseignements sur les événements anoxiques: Société Géologique du Nord, Publication, v. $25, x i+644$ p.

Bréhéret, J.-G., Caron, M., and Delamette, M., 1986, Niveau riches en matière organique dans l'Albien vocontien; quelques caractères du paléoenvironment; essai d'interprétation génétique: Documents du Bureau des Recherches Géologiques et Minières, v. 110, pp. 141-191.

Casey, R., 1961, The stratigraphical palaeontology of the Lower Greensand: Palaeontology, v. 3, pp. 487-621.

Collet, L.W., 1907, Sur quelques espèces de l'Albien Inférieur de Vöhrum (Hanovre): Mémoires de la Société de Physique et d'Histoire Naturelle de Genève, v. 35, pp. 519-529.

Colleté, C. (ed.), 2011, Stratotype Albien: Muséum national d'Histoire naturelle, Paris, Biotope Mèze, BRGM, Orléans, 446 p.

Hart, M.B., Amédro, F., and Owen, H.G., 1996, The Albian Stage and Substage boundaries: Bulletin de l'Institut Royal des Sciences Naturelles de Belgique, Sciences de la Terre, v. 66 (Supplement), pp. 45-56.

Herrle, J.O., 2002, Palaeoceanographic and palaeoclimatic implications of Mid-Cretaceous black shale formation in the Vocontian Basin and Atlantic: evidence from calcareous nannofossils and stable isotopes: Tübinger Mikropaläontologische Mitteilungen, v. 27, pp. 114.

Herrle, J.O., 2003, Reconstructing nutricline dynamics of mid-Cretaceous oceans: evidence from calcareous nannfossils from the Niveau Paquier black shale. Marine Micropalaeontology, v. 47, pp. 307-321.

Herrle, J.O., and Mutterlose, J., 2003, Calcareous nannofossils from Aptian-Lower Albian of southeast France: palaeoecological and biostratigraphic implications: Cretaceous Research, v. 24, pp. 1-22.

Herrle, J.O., Pross, J., Friedrich, O., and Hemleben, C., 2003, Short-term environmental changes in the Cretaceous Tethyan Ocean; micropalaeontological evidence from the early Albian Oceanic Anoxic Event 1b: Terra Nova, v. 15, pp. 14-19.

Herrle, J.O., Kößlere, P., Friedrich, O., Erlenkeuser, H., and Hemleben, C., 2004, High-resolution carbon isotope records of the Aptian to Lower Albian from SE France and the Mazagan Plateau (DSDP site 545): a stratigraphic tool for paleoceanographic and paleobiologic reconstruction: Earth and Planetary Science Letters, v. 218, pp. 149-161.

Huber, B.T., and Leckie, R.M., 2011, Planktic foraminiferal turnover across deep-sea Aptian/Albian boundary sections: Journal of Foraminiferal Research, v. 41, pp. 53-95.

Huber, B.T., MacLeod, K.G., Gröcke, D., and Kucera, M., 2011, Paleotemperature and paleosalinity inferences and chemostratigraphy across the Aptian/Albian boundary in the subtropical North Atlantic: Paleoceanography, v. 26, PA4221. doi:4210.1029/2011PA002178

Kennedy, W.J., Gale, A.S., Bown, P.R., Caron, M., Davey, R.J., Gröcke, D., and Wray, D.J., 2000, Integrated stratigraphy across the AptianAlbian boundary in the Marnes Bleues, at the Col de Pré-Guittard, Arnayon (Drôme), and at Tartonne (Alpes-de-Haute-Provence), France, a candidate Global boundary Stratotype Section and Point for the base of the Albian Stage: Cretaceous Research, v. 21, pp. 591-720. 
Kennedy, W.J., Gale, A.S., Huber, B.T., Petrizzo, Bown, P., Barchetta, A., and Jenkyns, H.C., 2014, Integrated stratigraphy across the Aptian/ Albian boundary at the Col de Pré-Guittard (southeast France): a candidate Global Boundary Stratotype Section: Cretaceous Research, v. 51, pp. 248-259.

Kuypers, M.M.M., Blokker, P., Erbacher, J., Kinkel, H., Pancost, R.D., Schouten, S., and Sinninghe Damsté, J.S., 2001, Massive expansion of marine Archaea during a mid-Cretaceous Anoxic Event: Science, v. 293, pp. 92-94.

Kuypers, M.M.M., Blokker, P., Hopmans, E.C., Kinkel, H., Pancost, R.D., Schouten, S., and Sinninghe Damsté, J.S., 2002, Archaeal remains dominate marine organic matter from early Albian oceanic anoxic event 1b: Palaeogeography, Palaeoclimatology, Palaeoecology, v. 185, pp. 211-234.

Leckie, R.M., Bralower, T.J., and Cashman, R., 2002, Oceanic anoxic events and plankton evolution: Biotic response to tectonic forcing during the mid-Cretaceous: Paleoceanography, v. 17, no. 3, pp. 13-1-13-29.

Orbigny, A.D., 1842-1843, Paléontologie française: terrains Crétacés, 2, Gastéropodes: Masson, Paris, 1-224 (1842); 225-456 (1843).

Petrizzo, M.R., Huber, B.T., Gale, A.S., Barchetta, A., and Jenkyns, H.C., 2012, Abrupt planktic foraminiferal turnover across the Niveau Kilian at Col de Pré-Guittard (Vocontian Basin, southeast France): new criteria for defining the Aptian/Albian boundary: Newsletters on Stratigraphy, v. 45, pp. 55-74.

Petrizzo, M.R., Huber, B.T., Gale, A.S., Barchetta, A., and Jenkyns, H.C., 2013, Erratum: Abrupt planktic foraminiferal turnover across the Niveau Kilian at Col de Pré-Guittard (Vocontian Basin, southeast France): new criteria for defining the Aptian/Albian boundary: Newsletters on Stratigraphy, v. 46, pp. 93.

Premoli Silva, I., 2010, Annual Report 2010 of the International Subcommission on Cretaceous Stratigraphy: Available at http://www2.mnhn. fr/hdt203/info/iscs

Premoli Silva, I., Caron, M., Leckie, R.M., Petrizzo, M.R., Soldan, D., and Verga, D., 2009, Paraticinella n. gen. and taxonomic revision of Ticinella bejaouaensis Sigal, 1966: Journal of Foraminiferal Research, v. 39, no. 2, pp. 126-137.

Rat, P., Magniez-Jannin, F., Chateuneuf, J.J., Damotte, R., Destombes, P., Fauconnier, P., Feuille, P., Manivit, P., Mogin, D., and Odin, G., 1979, Les stratotypes française 5. L'Albien de l'Aube: Centre National de la Recherche Scientifique, Paris, $446 \mathrm{p}$.

Roth, P.H., 1978, Cretaceous nannoplankton biostratigraphy and oceanography of the northwestern Atlantic Ocean. in Benson, W.E., Sheridan, R.E. et al. (eds.), Initial Reports of the Deep Sea Drilling Project: U.S. Government Printing Office, Washington, D.C., v. 44, pp. 731-760.

Schlotheim, E.F. von, 1813, Beiträge zur Naturgeschichte der Versteinerungen in geognostischer Hinsicht: Leonard's Taschenbuch für die gessamte Mineralogie, v. 7, pp. 3-134.

Seyed-Emami, K., and Wilmsen, M., 2016, Leymeriellidae (Cretaceous ammonites) from the lower Albian of Esfahan and Khur (Central Iran): Cretaceous Research, v. 60, pp. 78-90.

Sissingh, W., 1977, Biostratigraphy of Cretaceous calcareous nannoplankton: Geologie en Mijnbouw, v. 56, pp. 37-50.

Stover, L.E., 1966, Cretaceous coccoliths and associated nannofossils from France and the Netherlands: Micropaleontology, v. 12, pp. 133-167.

Trabucho Alexandre, J.T., van Gilst, R.I., Rodriguez-Lopez, J.P., and de Boer, P.L., 2011, The sedimentary expression of oceanic anoxic event $1 \mathrm{~b}$ in the North Atlantic: Sedimentology, v. 58, pp. 1217-1246.

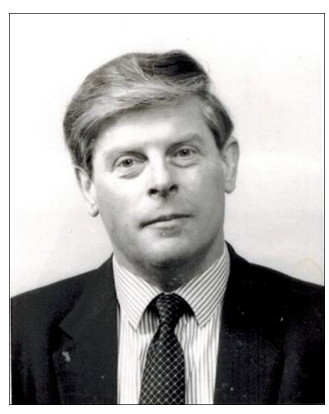

Jim Kennedy is an Emeritus Professor of Natural History, and a Director Emeritus of the Oxford University Museum of Natural History. Current research is concentrated on the systematics and biostratigraphy of Cretaceous ammonite faunas from the United Kingdom, Germany, Austria, Uzbekistan, Kazakhstan, Algeria, Tunisia, Nigeria, KwaZulu-Natal in South Africa, Tanzania and Colombia.

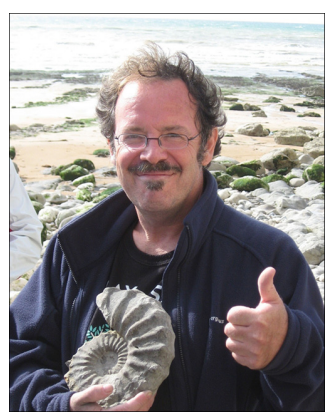

Andy Gale is a Professor of Geology in the Department of Earth and Environmental Sciences at the University of Portsmouth. His current research interests include Chalk stratigraphy, sedimentation and diagenesis, the systematics and evolution of pedunculated cirripedes, and the systematics and the stratigraphic distribution of Cretaceous microcrinoids.

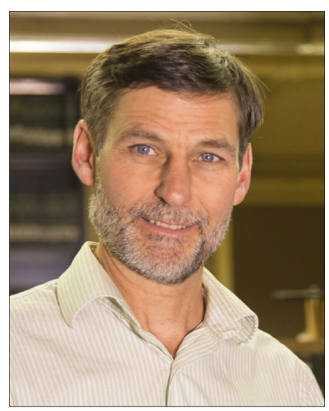

Brian Huber is a Curator of Foraminifera at the Smithsonian's National Museum of Natural History's Department of Paleobiology and is Vice-Chair of the International Commission on Stratigraphy. His research focuses on changes in global climate and between 115 and 35 million years ago and the evolutionary dynamics and extinction of Cretaceous and Paleogene planktonic foraminifera during that time interval.

Maria Rose Petrizzo is an Associate Professor at the Department of Earth Sciences, University of Milano (Italy), Chair of the Subcommission on Cretaceous Stratigraphy and Secretary of the Subcommission on Stratigraphic Classification of the International Commission on Stratigraphy. Her research interests are focused on Cretaceous and Paleogene planktonic foraminifera with emphasis on biotic changes during times of environmental perturbations, and on the revision of the Cretaceous planktonic foraminifera taxonomy, biostratigraphy and biozonation for updating the Geological Time Scale and for the definition of the GSSPs.

Paul Bown is a Professor of Micropalaeontology in the Department of Earth Sciences at University College London. His research has predominantly focussed on the palaeobiology and geological applications of fossil marine plankton, known as coccolithophores (or more broadly calcareous nannofossils) and in particular the relationship between environmental change and evolution. This research is underpinned by extensive stratigraphic and taxonomic work and he has described more than 150 new Mesozoic and Paleogene nannofossil taxa.

Hugh C. Jenkyns is a Professor of Stratigraphy in the Department of Earth Sciences at Oxford University. His fields of expertise range across geochemistry (Li, C, N, O, S, Fe, Sr, Mo, Nd, U isotopes), sedimentology and stratigraphy, particularly of Jurassic and Cretaceous sediments. Research highlights include studies on the causes and consequences of 'oceanic anoxic events' (a term coined in a joint paper published in 1976) and the application of chemostratigraphy to understanding Mesozoic palaeoceanography and climate change. 